\title{
Some Remarks on Quasi-Generalized CR-Null Geometry in Indefinite Nearly Cosymplectic Manifolds
}

\author{
Fortuné Massamba and Samuel Ssekajja \\ School of Mathematics, Statistics and Computer Science, University of KwaZulu-Natal, Private Bag X01, Scottsville 3209, South Africa \\ Correspondence should be addressed to Fortuné Massamba; massfort@yahoo.fr
}

Received 12 August 2016; Accepted 11 October 2016

Academic Editor: Luc Vrancken

Copyright ( 2016 F. Massamba and S. Ssekajja. This is an open access article distributed under the Creative Commons Attribution License, which permits unrestricted use, distribution, and reproduction in any medium, provided the original work is properly cited.

Attention is drawn to some distributions on ascreen Quasi-Generalized Cauchy-Riemannian (QGCR) null submanifolds in an indefinite nearly cosymplectic manifold. We characterize totally umbilical and irrotational ascreen QGCR-null submanifolds. We finally discuss the geometric effects of geodesity conditions on such submanifolds.

\section{Introduction}

One of the current interesting research areas in semiRiemannian geometry is the theory of null (or lightlike) submanifolds. An intrinsic approach to the theory of null submanifolds was advanced by Kupeli [1], yet an extrinsic counterpart had to wait for Duggal and Bejancu [2], and later by Duggal and Sahin [3]. Since then, many researchers have laboured to extend their theories with evidence from the following few selected papers: [3-11] and other references therein. The rapid increase in research on this topic, since 1996, is inspired by the numerous applications of the theory to mathematical physics, particularly in general relativity. More precisely, in general relativity, null submanifolds represent different models of black hole horizons (see $[2,3]$ for details).

In [5], the authors initiated the study of generalized CR(GCR-) null submanifolds of an indefinite Sasakian manifold, which are tangent to the structure vector field, $\xi$, of the almost contact structure $(\bar{\phi}, \xi, \eta)$. Moreover, when $\xi$ is tangent to the submanifold, Calin [12] proved that it belongs to its screen distribution. This assumption is widely accepted and it has been applied in many papers on null contact geometry, for instance, $[3,5,8-11,13]$. It is worth mentioning that $\xi$ is a global vector field defined on the entire tangent bundle of the ambient almost contact manifold. Thus, restricting it to the screen distribution is only one of those cases in which it can be placed. In the study of Riemannian CR-submanifolds of
Sasakian manifolds, Yano and Kon [14, p. 43] proved that making $\xi$ a normal vector field in such scenario leads to an anti-invariant submanifold, and hence $\xi$ was kept tangent to the CR-submanifold. Their proof leans against the fact that the shape operator on such CR-submanifold is naturally symmetric with respect to the induced Riemannian metric $g$. On the other hand, the shape operators of any $r$-null submanifold are generally not symmetric with respect to the induced degenerate metric $g$ (see $[2,3]$ for details).

In an attempt to generalize $\xi$, we introduced a special class of CR-null submanifolds of a nearly Sasakian manifold, known as quasi-generalized CR- (QGCR-) null submanifold [15], for which the classical GCR-null submanifolds [3] form part. Among other benefits, generalizing $\xi$ leads to QGCR-null submanifolds of lower dimensions and with quite different geometric properties compared to respective GCRnull submanifolds.

The purpose of this paper is to investigate the geometry of distributions on ascreen QGCR-null submanifolds of indefinite nearly cosymplectic manifolds. A null submanifold $M$ of an indefinite nearly cosymplectic manifold is called ascreen if the structure vector field $\xi$ belongs to $\operatorname{Rad}(T M) \oplus$ $l \operatorname{tr}(T M)$ [16]. The paper is organized as follows. In Section 2, we present the basic notions of null submanifolds and nearly cosymplectic manifolds. More details can be found in [1722]. In Section 3, we review the basic notions of QGCR-null submanifolds and we give an example of ascreen QGCR-null 
submanifold. In Section 4, we discuss totally umbilical, totally geodesic, and irrotational ascreen QGCR-null submanifolds of an indefinite nearly cosymplectic space form $\bar{M}(\bar{c})$. Finally, in Section 5 we investigate the geodesity of the distributions $D$ and $\widehat{D}$.

\section{Preliminaries}

Let $M^{m}$ be a codimension $n$ submanifold of a semiRiemannian manifold $(\bar{M}, \bar{g})$ of constant index $v, 1 \leq v \leq$ $m+n$, with $m, n \geq 1$. Then, $M$ is said to be a null submanifold of $\bar{M}$ if the tangent and normal bundles of $M$ have a nontrivial intersection. This intersection defines a smooth distribution on $M$, called the radical distribution [2]. More precisely, consider $p \in M$; one defines the orthogonal complement $T_{p} M^{\perp}$ of the tangent space $T_{p} M$ by

$$
T_{p} M^{\perp}=\left\{X \in T_{p} M: \bar{g}(X, Y)=0, \forall Y \in T_{p} M\right\} .
$$

If we denote the radical distribution on $M$ by $\operatorname{Rad} T_{p} M$, then $\operatorname{Rad} T_{p} M=\operatorname{Rad} T_{p} M^{\perp}=T_{p} M \cap T_{p} M^{\perp}$. The submanifold $M$ of $\bar{M}$ is said to be $r$-null submanifold (one supposes that the index of $\bar{M}$ is $\nu \geq r$ ), if the mapping $\operatorname{Rad} T M: p \in M \rightarrow$ $\operatorname{Rad} T_{p} M$ defines a smooth distribution on $M$ of rank $r>0$.

In this paper, $r$-null submanifold will simply be called a null submanifold and $g=\left.\bar{g}\right|_{T M}$ is a null metric, unless we need to specify $r$.

Let $S(T M)$ be a screen distribution which is a semiRiemannian complementary distribution of $\operatorname{Rad} T M$ in $T M$; that is,

$$
T M=\operatorname{Rad} T M \perp S(T M) .
$$

Consider a screen transversal bundle $S\left(T M^{\perp}\right)$, which is semiRiemannian and complementary to $\operatorname{Rad} T M$ in $T M^{\perp}$. For any local basis $\left\{E_{1}, \ldots, E_{r}\right\}$ of $\operatorname{Rad} T M$, there exists a local null frame $\left\{N_{1}, \ldots, N_{r}\right\} \subset S\left(T M^{\perp}\right)$ in $S(T M)^{\perp}$ such that $g\left(E_{i}, N_{j}\right)=\delta_{i j}$ and $\bar{g}\left(N_{i}, N_{j}\right)=0$. It follows that there exists a null transversal vector bundle $l \operatorname{tr}(T M)$ locally spanned by $\left\{N_{1}, \ldots, N_{r}\right\}$ (see details in $[2,3]$ ). If $\operatorname{tr}(T M)$ denotes the complementary (but not orthogonal) vector bundle to $T M$ in $T \bar{M}$, then,

$$
\begin{aligned}
\operatorname{tr}(T M) & =l \operatorname{tr}(T M) \perp S\left(T M^{\perp}\right), \\
T \bar{M} & =S(T M) \perp S\left(T M^{\perp}\right) \\
& \perp\{\operatorname{Rad} T M \oplus l \operatorname{tr}(T M)\} .
\end{aligned}
$$

It is important to note that the screen distribution $S(T M)$ is not unique and is canonically isomorphic to the factor vector bundle TM/RadTM [1].

Given a null submanifold $M$, then the following classifications of $M$ are well-known [2]: (i) $M$ is $r$-null if $1 \leq r<$ $\min \{m, n\}$; (ii) $M$ is coisotropic if $1 \leq r=n<m, S\left(T M^{\perp}\right)=$ $\{0\}$; (iii) $M$ is isotropic if $1 \leq r=m<n, S(T M)=\{0\}$; (iv) $M$ is totally null if $r=n=m, S(T M)=S\left(T M^{\perp}\right)=\{0\}$.
Where necessary, the following range of indices will be used

$$
\begin{gathered}
i, j, k \in\{1, \ldots, r\}, \\
\alpha, \beta, \gamma \in\{r+1, \ldots, n\} .
\end{gathered}
$$

Consider a local quasi-orthonormal fields of frames of $\bar{M}$ along $M$ as

$$
\left\{E_{1}, \ldots, E_{r}, N_{1}, \ldots, N_{r}, X_{r+1}, \ldots, X_{m}, W_{1+r}, \ldots, W_{n}\right\}
$$

where $\left\{X_{r+1}, \ldots, X_{m}\right\}$ and $\left\{W_{1+r}, \ldots, W_{n}\right\}$ are, respectively, orthonormal bases of $\Gamma(S(T M))$ and $\Gamma\left(S\left(T M^{\perp}\right)\right)$.

Throughout the paper we consider $\Gamma(\Xi)$ to be a set of smooth sections of the vector bundle $\Xi$.

Let $P$ be the projection morphism of TM onto $S(T M)$. Then, the following are the Gauss-Weingarten equations of $r$-null submanifold $M$ and $S(T M)$ (see $[2,3]$ for detailed explanations):

$$
\begin{gathered}
\bar{\nabla}_{X} Y=\nabla_{X} Y+\sum_{i=1}^{r} h_{i}^{l}(X, Y) N_{i}+\sum_{\alpha=r+1}^{n} h_{\alpha}^{s}(X, Y) W_{\alpha}, \\
\bar{\nabla}_{X} N_{i}=-A_{N_{i}} X+\sum_{j=1}^{r} \tau_{i j}(X) N_{j}+\sum_{\alpha=r+1}^{n} \rho_{i \alpha}(X) W_{\alpha}, \\
\bar{\nabla}_{X} W_{\alpha}=-A_{W_{\alpha}} X+\sum_{i=1}^{r} \varphi_{\alpha i}(X) N_{i}+\sum_{\beta=r+1}^{n} \sigma_{\alpha \beta}(X) W_{\beta}, \\
\nabla_{X} P Y=\nabla_{X}^{*} P Y+\sum_{i=1}^{r} h_{i}^{*}(X, P Y) E_{i}, \\
\nabla_{X} E_{i}=-A_{E_{i}}^{*} X-\sum_{j=1}^{r} \tau_{j i}(X) E_{j}, \quad \forall X, Y \in \Gamma(T M),
\end{gathered}
$$

where $\nabla$ and $\nabla^{*}$ are the induced connections on $T M$ and $S(T M)$, respectively and $h_{i}^{l}$ and $h_{\alpha}^{s}$ are symmetric bilinear forms known as local null and screen fundamental forms of $T M$, respectively. Furthermore, $h_{i}^{*}$ are the second fundamental forms of $S(T M) . A_{N_{i}}, A_{E_{i}}^{*}$, and $A_{W_{\alpha}}$ are linear operators on $T M$ while $\tau_{i j}, \rho_{i \alpha}, \varphi_{\alpha i}$, and $\sigma_{\alpha \beta}$ are 1-forms on TM. Note that the second fundamental tensor of $M$ is given by

$$
h(X, Y)=\sum_{i=1}^{r} h_{i}^{l}(X, Y) N_{i}+\sum_{\alpha=r+1}^{n} h_{\alpha}^{s}(X, Y) W_{\alpha},
$$

for any $X, Y \in \Gamma(T M)$. The connection $\nabla^{*}$ is a metric connection on $S(T M)$ while $\nabla$ is generally not a metric connection and is given by

$$
\begin{aligned}
& \left(\nabla_{X} g\right)(Y, Z) \\
& \quad=\sum_{i=1}^{r}\left\{h_{i}^{l}(X, Y) \lambda_{i}(Z)+h_{i}^{l}(X, Z) \lambda_{i}(Y)\right\},
\end{aligned}
$$

for any $X, Y \in \Gamma(T M)$ and $\lambda_{i}$ are 1-forms given by $\lambda_{i}(X)=$ $\bar{g}\left(X, N_{i}\right)$, for all $X \in \Gamma(T M)$. By using (7), (8), and (9), the 
curvature tensors $\bar{R}$ and $R$ of $\bar{M}$ and $M$, respectively, are related as, for any $X, Y, Z, W \in \Gamma(T M)$,

$$
\begin{aligned}
\bar{R}(X, W, Z, Y)= & \bar{g}(R(X, W) Z, Y) \\
& +\bar{g}\left(A_{h^{l}(X, Z)} W, Y\right) \\
& -\bar{g}\left(A_{h^{l}(W, Z)} X, Y\right) \\
& +\bar{g}\left(A_{h^{s}(X, Z)} W, Y\right) \\
& -\bar{g}\left(A_{h^{s}(W, Z)} X, Y\right) \\
& +\bar{g}\left(\left(\nabla_{X} h^{l}\right)(W, Z), Y\right) \\
& -\bar{g}\left(\left(\nabla_{W} h^{l}\right)(X, Z), Y\right) \\
& +\bar{g}\left(D^{l}\left(X, h^{s}(W, Z)\right), Y\right) \\
& -\bar{g}\left(D^{l}\left(W, h^{s}(X, Z)\right), Y\right) \\
& +\bar{g}\left(\left(\nabla_{X} h^{s}\right)(W, Z), Y\right) \\
& -\bar{g}\left(\left(\nabla_{W} h^{s}\right)(X, Z), Y\right) \\
& +\bar{g}\left(D^{s}\left(X, h^{l}(W, Z)\right), Y\right) \\
& -\bar{g}\left(D^{s}\left(W, h^{l}(X, Z)\right), Y\right) .
\end{aligned}
$$

A null submanifold $(M, g)$ of an indefinite manifold $(\bar{M}, \bar{g})$ is said to be totally umbilical in $\bar{M}$ [3] if there is a smooth transversal vector field $\mathscr{H} \in \Gamma(\operatorname{tr}(T M))$, called the transversal curvature vector of $M$ such that

$$
h=g \otimes \mathscr{H} .
$$

Moreover, it is easy to see that $M$ is totally umbilical in $\bar{M}$, if and only if on each coordinate neighborhood $U$ there exist smooth vector fields $\mathscr{H}^{l} \in \Gamma(\operatorname{ltr}(T M))$ and $\mathscr{H}^{s} \in$ $\Gamma\left(S\left(T M^{\perp}\right)\right)$ and smooth functions $\mathscr{H}_{i}^{l} \in F(\operatorname{lt}(T M))$ and $\mathscr{H}_{\alpha}^{s} \in F\left(S\left(T M^{\perp}\right)\right)$ such that,

$$
\begin{aligned}
h^{l}(X, Y) & =\mathscr{H}^{l} g(X, Y), \\
h^{s}(X, Y) & =\mathscr{H}^{s} g(X, Y), \\
h_{i}^{l}(X, Y) & =\mathscr{H}_{i}^{l} g(X, Y), \\
h_{\alpha}^{s}(X, Y) & =\mathscr{H}_{\alpha}^{s} g(X, Y),
\end{aligned}
$$

for all $X, Y \in \Gamma(T M)$.

Let us now consider $\bar{M}$ to be a $(2 n+1)$-dimensional manifold endowed with an almost contact structure $(\bar{\phi}, \xi, \eta)$; that is, $\bar{\phi}$ is a tensor field of type $(1,1), \xi$ is a vector field, and $\eta$ is a 1 -form satisfying

$$
\begin{aligned}
\bar{\phi}^{2} & =-\llbracket+\eta \otimes \xi, \\
\eta(\xi) & =1,
\end{aligned}
$$

$$
\begin{aligned}
\eta \circ \bar{\phi} & =0, \\
\bar{\phi}(\xi) & =0 .
\end{aligned}
$$

Then $(\bar{\phi}, \xi, \eta, \bar{g})$ is called an indefinite almost contact metric structure on $\bar{M}$ if $(\bar{\phi}, \xi, \eta)$ is an almost contact structure on $\bar{M}$ and $\bar{g}$ is a semi-Riemannian metric on $\bar{M}$ such that [19], for any vector field $\bar{X}, \bar{Y}$ on $\bar{M}$,

$$
\begin{aligned}
& \bar{g}(\bar{\phi} \bar{X}, \bar{\phi} \bar{Y})=\bar{g}(\bar{X}, \bar{Y})-\eta(\bar{X}) \eta(\bar{Y}), \\
& \eta(\bar{X})=\bar{g}(\xi, \bar{X}) .
\end{aligned}
$$

An indefinite almost contact metric manifold $(\bar{M}, \bar{\phi}, \xi, \eta, \bar{g})$ is said to be nearly cosymplectic if

$$
(\bar{\nabla} \bar{X} \bar{\phi}) \bar{Y}+(\bar{\nabla} \bar{Y} \bar{\phi}) \bar{X}=0, \quad \forall \bar{X}, \bar{Y} \in \Gamma(T \bar{M}),
$$

where $\bar{\nabla}$ is the Levi-Civita connection for $\bar{g}$. Taking $\bar{Y}=\xi$ in (19), we get

$$
\bar{\nabla}_{\bar{X}} \xi=-\bar{H} \bar{X}, \quad \forall \bar{X} \in \Gamma(T \bar{M}) .
$$

It is easy to verify the following properties of $\bar{H}$ :

$$
\begin{aligned}
\bar{H} \bar{\phi}+\bar{\phi} \bar{H} & =0, \\
\bar{H} \xi & =0, \\
\eta \circ \bar{H} & =0, \\
\left(\bar{\nabla}_{X} \bar{\phi}\right) \xi & =\bar{\phi} \bar{H} X, \\
\bar{g}(\bar{H} \bar{X}, \bar{Y}) & =-\bar{g}(\bar{X}, \bar{H} \bar{Y})
\end{aligned}
$$

(i.e., $\bar{H}$ is skew-symmetric),

for all $\bar{X}, \bar{Y} \in \Gamma(T \bar{M})$. Let $\Omega$ denote the fundamental 2-form of $\bar{M}$ defined by

$$
\Omega(\bar{X}, \bar{Y})=\bar{g}(\bar{X}, \bar{\phi} \bar{Y}), \quad \bar{X}, \bar{Y} \in \Gamma(T \bar{M}) .
$$

Then the 1 -form $\eta$ and tensor $\bar{H}$ are related as follows.

Lemma 1. Let $(\bar{M}, \bar{\phi}, \xi, \eta, \bar{g})$ be indefinite nearly cosymplectic. Then,

$$
d \eta(\bar{X}, \bar{Y})=\bar{g}(\bar{X}, \bar{H} \bar{Y}), \quad \forall \bar{X}, \bar{Y} \in \Gamma(T \bar{M}) .
$$

Moreover, $\bar{M}$ is cosymplectic if and only if $\bar{H}$ vanishes identically on $\bar{M}$.

Note that, for all $\bar{X}, \bar{Y}, \bar{Z} \in \Gamma(T \bar{M})$,

$$
\bar{g}((\bar{\nabla} \bar{Z} \bar{\phi}) \bar{X}, \bar{Y})=-\bar{g}\left(\bar{X},\left(\bar{\nabla}_{\bar{Z}} \bar{\phi}\right) \bar{Y}\right),
$$

which means that the tensor $\bar{\nabla} \bar{\phi}$ is skew-symmetric. The following lemma is fundamental to the sequel. 
Lemma 2. Let $\bar{M}$ be a nearly cosymplectic manifold. Then

$$
\begin{aligned}
\left(\bar{\nabla}_{\bar{X}} \bar{\phi}\right) \bar{\phi} \bar{Y}= & -\bar{\phi}\left(\bar{\nabla}_{\bar{X}} \bar{\phi}\right) \bar{Y}-\bar{g}(\bar{Y}, \bar{H} \bar{X}) \xi \\
& -\eta(\bar{Y}) \bar{H} \bar{X}, \\
\left(\bar{\nabla}_{\bar{\phi} \bar{X}} \bar{\phi}\right) \bar{\phi} \bar{Y}= & -\left(\bar{\nabla}_{\bar{X}} \bar{\phi}\right) \bar{Y}-\eta(\bar{X}) \bar{\phi} \bar{H} \bar{Y} \\
& +\eta(\bar{Y}) \bar{\phi} \bar{H} \bar{X},
\end{aligned}
$$

for all $\bar{X}, \bar{Y} \in \Gamma(T \bar{M})$.

Proof. The proof follows from a straightforward calculation.

\section{Quasi-Generalized CR-Null Submanifolds}

We recall some basic notions on QGCR-null submanifolds (see [15] for details).

The structure vector field $\xi$ of an indefinite almost contact manifold $(\bar{M}, \bar{g})$ can be written according to decomposition (4) as follows:

$$
\xi=\xi_{S}+\sum_{i=1}^{r} a_{i} E_{i}+\sum_{i=1}^{r} b_{i} N_{i}+\sum_{\alpha=r+1}^{n} c_{\alpha} W_{\alpha}
$$

where $\xi_{S}$ is a smooth vector field of $S(T M)$ while $a_{i}=\eta\left(N_{i}\right)$, $b_{i}=\eta\left(E_{i}\right)$, and $c_{\alpha}=\epsilon_{\alpha} \eta\left(W_{\alpha}\right)$, all smooth functions on $\bar{M}$. Here $\epsilon_{\alpha}=\bar{g}\left(W_{\alpha}, W_{\alpha}\right)$.

We adopt the definition of quasi-generalized CR(QGCR-) null submanifolds given in [15] for indefinite nearly cosymplectic manifolds.

Definition 3. Let $\left(M, g, S(T M), S\left(T M^{\perp}\right)\right)$ be a null submanifold of an indefinite nearly cosymplectic manifold $(\bar{M}, \bar{g})$. We say that $M$ is quasi-generalized CR- (QGCR-) null submanifold of $\bar{M}$ if the following conditions are satisfied.

(i) There exist two distributions $D_{1}$ and $D_{2}$ of $\operatorname{RadTM}$ such that

$$
\begin{aligned}
\operatorname{Rad} T M & =D_{1} \oplus D_{2}, \\
\bar{\phi} D_{1} & =D_{1}, \\
\bar{\phi} D_{2} & \subset S(T M) .
\end{aligned}
$$
that

(ii) There exist vector bundles $D_{0}$ and $\bar{D}$ over $S(T M)$ such

$$
\begin{aligned}
S(T M)=\left\{\bar{\phi} D_{2} \oplus \bar{D}\right\} & \perp D_{0}, \\
& \text { with } \bar{\phi} D_{0} \subseteq D_{0}, \bar{D}=\bar{\phi} \mathcal{S} \oplus \bar{\phi} \mathscr{L},
\end{aligned}
$$

where $D_{0}$ is a nondegenerate distribution on $M$ and $\mathscr{L}$ and $\mathcal{S}$ are, respectively, vector subbundles of $l \operatorname{tr}(T M)$ and $S\left(T M^{\perp}\right)$.

If $D_{1} \neq\{0\}, D_{0} \neq\{0\}, D_{2} \neq\{0\}$, and $\mathcal{S} \neq\{0\}$, then $M$ is called a proper $Q G C R$-null submanifold.

A proof of the following proposition uses similar arguments as in [15].
Proposition 4. A QGCR-null submanifold $M$ of an indefinite nearly cosymplectic manifold $\bar{M}$ tangent to the structure vector field $\xi$ is a GCR-null submanifold.

Using (2), the tangent bundle of any QGCR-null submanifold, TM, can be decomposed as

$$
\begin{aligned}
& T M=D \oplus \widehat{D}, \\
& \text { with } D=D_{0} \perp D_{1}, \widehat{D}=\left\{D_{2} \perp \bar{\phi} D_{2}\right\} \oplus \bar{D} .
\end{aligned}
$$

Unlike a GCR-null submanifold, in a QGCR-null submanifold, $D$ is invariant with respect to $\bar{\phi}$ while $\widehat{D}$ is not generally anti-invariant.

Throughout this paper, we suppose that $(M, g, S(T M)$, $S\left(T M^{\perp}\right)$ ) is a proper QGCR-null submanifold. From the above definition, we can easily deduce the following:

(1) Condition (i) implies that $\operatorname{dim}(\operatorname{Rad} T M)=s \geq 3$.

(2) Condition (ii) implies that $\operatorname{dim}(D) \geq 4 l \geq 4$ and $\operatorname{dim}\left(D_{2}\right)=\operatorname{dim}(\mathscr{L})$.

Definition 5 (see [16]). A null submanifold $M$ of a semiRiemannian manifold $\bar{M}$ is said to be ascreen if the structure vector field, $\xi$, belongs to $\operatorname{Rad} T M \oplus l \operatorname{tr}(T M)$.

From Definition 3.3, Lemma 3.6, and Theorem 3.7 of [15], we have the following.

Theorem 6. Let $\left(M, g, S(T M), S\left(T M^{\perp}\right)\right)$ be an ascreen QGCR-null submanifold of an indefinite nearly cosymplectic manifold $\bar{M}$; then $\xi \in \Gamma\left(D_{2} \oplus \mathscr{L}\right)$. If $M$ is a 3-null $Q G C R$ submanifold of an indefinite nearly cosymplectic manifold $(\bar{M}$, $\bar{g})$, then $M$ is ascreen null submanifold if and only if $\bar{\phi} \mathscr{L}=$ $\bar{\phi} D_{2}$.

Proof. The proof follows from straightforward calculation as in [15].

It is crucial to note the following aspects with ascreen QGCR-null submanifold: item (2) of Definition 3 implies that $\operatorname{dim}(D) \geq 4 l \geq 4$ and $\operatorname{dim}\left(D_{2}\right)=\operatorname{dim}(\mathscr{L})$. Thus $\operatorname{dim}(M) \geq 7$ and $\operatorname{dim}(\bar{M}) \geq 11$, and any 7-dimensional ascreen QGCRnull submanifold is 3-null.

In what follows, we construct an ascreen QGCR-null submanifold of a special nearly cosymplectic manifold with $\bar{H}=$ 0 (i.e., $\bar{M}$ is a cosymplectic manifold). Thus, let $\left(\mathbb{R}_{q}^{2 m+1}, \bar{\phi}_{0}\right.$, $\xi, \eta, \bar{g})$ denote the manifold $\mathbb{R}_{q}^{2 m+1}$ with its usual cosymplectic structure given by

$$
\begin{aligned}
& \eta=d z, \\
& \xi=\partial z, \\
& \bar{g} \\
& \quad=\eta \otimes \eta-\sum_{i=1}^{q / 2}\left(d x^{i} \otimes d x^{i}+d y^{i} \otimes d y^{i}\right)
\end{aligned}
$$




$$
\begin{array}{r}
+\sum_{i=q+1}^{m}\left(d x^{i} \otimes d x^{i}+d y^{i} \otimes d y^{i}\right), \\
\phi_{0}\left(\sum_{i=1}^{m}\left(X_{i} \partial x^{i}+Y_{i} \partial y^{i}\right)+Z \partial z\right) \\
=\sum_{i=1}^{m}\left(Y_{i} \partial x^{i}-X_{i} \partial y^{i}\right),
\end{array}
$$

where $\left(x^{i}, y^{i}, z\right)$ are Cartesian coordinates and $\partial t_{k}=\partial / \partial t^{k}$, for $t \in \mathbb{R}^{2 m+1}$.

Now, we use the above structure to construct the following example.

Example 7. Let $\bar{M}=\left(\mathbb{R}_{4}^{11}, \bar{g}\right)$ be a semi-Euclidean space, with $\bar{g}$ being of signature $(-,-,+,+,+,-,-,+,+,+,+)$ with respect to the canonical basis

$$
\left(\partial x_{1}, \partial x_{2}, \partial x_{3}, \partial x_{4}, \partial x_{5}, \partial y_{1}, \partial y_{2}, \partial y_{3}, \partial y_{4}, \partial y_{5}, \partial z\right)
$$

Let $(M, g)$ be a submanifold of $\bar{M}$ given by

$$
\begin{aligned}
x^{1} & =y^{4}, \\
y^{1} & =-x^{4}, \\
z & =x^{2} \sin \theta+y^{2} \cos \theta, \\
y^{5} & =\left(x^{5}\right)^{1 / 2},
\end{aligned}
$$

where $\theta \in(0, \pi / 2)$. By direct calculations, we can see that the vector fields

$$
\begin{aligned}
& E_{1}=\partial x_{4}+\partial y_{1}, \\
& E_{2}=\partial x_{1}-\partial y_{4}, \\
& E_{3}=\sin \theta \partial x_{2}+\cos \theta \partial y_{2}+\partial z, \\
& X_{1}=2 y^{5} \partial x_{5}+\partial y_{5}, \\
& X_{2}=-\cos \theta \partial x_{2}+\sin \theta \partial y_{2}, \\
& X_{3}=\partial y_{3}, \\
& X_{4}=\partial x_{3}
\end{aligned}
$$

form a local frame of $T M$. Then $\operatorname{Rad} T M$ is spanned by $\left\{E_{1}, E_{2}, E_{3}\right\}$, and, therefore, $M$ is 3-null. Further, $\bar{\phi}_{0} E_{1}=E_{2}$; therefore we set $D_{1}=\operatorname{Span}\left\{E_{1}, E_{2}\right\}$. Also $\bar{\phi}_{0} E_{3}=-X_{2}$ and thus $D_{2}=\operatorname{Span}\left\{E_{3}\right\}$. It is easy to see that $\bar{\phi}_{0} X_{3}=X_{4}$, so we set $D_{0}=\operatorname{Span}\left\{X_{3}, X_{4}\right\}$. On the other hand, following direct calculations, we have

$$
\begin{aligned}
& N_{1}=\frac{1}{2}\left(\partial x_{4}-\partial y_{1}\right) \\
& N_{2}=\frac{1}{2}\left(-\partial x_{1}-\partial y_{4}\right),
\end{aligned}
$$

$$
\begin{aligned}
N_{3} & =\frac{1}{2}\left(-\sin \theta \partial x_{2}-\cos \theta \partial y_{2}+\partial z\right), \\
W & =\partial x_{5}-2 y^{5} \partial y_{5},
\end{aligned}
$$

from which $l \operatorname{tr}(T M)=\operatorname{Span}\left\{N_{1}, N_{2}, N_{3}\right\}$ and $S\left(T M^{\perp}\right)=$ $\operatorname{Span}\{W\}$. Clearly, $\bar{\phi}_{0} N_{2}=-N_{1}$. Further, $\bar{\phi}_{0} N_{3}=(1 / 2) X_{2}$ and thus $\mathscr{L}=\operatorname{Span}\left\{N_{3}\right\}$. Notice that $\bar{\phi}_{0} N_{3}=-(1 / 2) \bar{\phi}_{0} E_{3}$ and therefore $\bar{\phi}_{0} \mathscr{L}=\bar{\phi}_{0} D_{2}$. Also, $\bar{\phi}_{0} W=-X_{1}$ and therefore $\mathcal{S}=$ $\operatorname{Span}\{W\}$. Finally, we calculate $\xi$ as follows. Using Theorem 6 we have $\xi=a E_{3}+b N_{3}$. Applying $\bar{\phi}_{0}$ to this equation we obtain $a \bar{\phi}_{0} E_{3}+b \bar{\phi}_{0} N_{3}=0$. Now, substituting for $\bar{\phi}_{0} E_{3}$ and $\bar{\phi}_{0} N_{3}$ in this equation we get $2 a=b$, from which we get $\xi=$ $(1 / 2)\left(E_{3}+2 N_{3}\right)$. Since $\bar{\phi}_{0} \xi=0$ and $\bar{g}(\xi, \xi)=1$, we conclude that $(M, g)$ is an ascreen QGCR-null submanifold of $\bar{M}$.

Proposition 8. There exist no coisotropic, isotropic, or totally null proper QGCR-null submanifolds of an indefinite nearly cosymplectic manifold.

\section{Umbilical and Geodesic Ascreen QGCR-Null Submanifolds}

In this section, we prove two main theorems concerning totally umbilical, totally geodesic and irrotational ascreen QGCR-null submanifolds of $\bar{M}$. An indefinite nearly cosymplectic manifold $\bar{M}$ is called an indefinite nearly cosymplectic space form, denoted by $\bar{M}(\bar{c})$, if it has the constant $\bar{\phi}$-sectional curvature $\bar{c}$. The curvature tensor $\bar{R}$ of the indefinite nearly cosymplectic space form $\bar{M}(\bar{c})$ is given by [21]:

$$
\begin{aligned}
4 \bar{R} & (\bar{X}, \bar{W}, \bar{Z}, \bar{Y})=\bar{g}((\bar{\nabla} \bar{W} \bar{\phi}) \bar{Z},(\bar{\nabla} \bar{X} \bar{\phi}) \bar{Y}) \\
& -\bar{g}((\bar{\nabla} \bar{W} \bar{\phi}) \bar{Y},(\bar{\nabla} \bar{X} \bar{\phi}) \bar{Z}) \\
& -2 \bar{g}\left((\bar{\nabla} \bar{W} \bar{\phi}) \bar{X},\left(\bar{\nabla}_{\bar{Y}} \bar{\phi}\right) \bar{Z}\right)+\bar{g}(\bar{H} \bar{W}, \bar{Z}) \\
& \cdot \bar{g}(\bar{H} \bar{X}, \bar{Y})-\bar{g}(\bar{H} \bar{W}, \bar{Y}) \bar{g}(\bar{H} \bar{X}, \bar{Z}) \\
& -2 \bar{g}(\bar{H} \bar{W}, \bar{X}) \bar{g}(\bar{H} \bar{Y}, \bar{Z})-\eta(\bar{W}) \eta(\bar{Y}) \\
& -\bar{g}(\bar{H} \bar{X}, \bar{H} \bar{Z})+\eta(\bar{W}) \eta(\bar{Z}) \bar{g}(\bar{H} \bar{X}, \bar{H} \bar{Y}) \\
& +\eta(\bar{X}) \eta(\bar{Y}) \bar{g}(\bar{H} \bar{W}, \bar{H} \bar{Z})-\eta(\bar{X}) \eta(\bar{Z}) \\
& \cdot \bar{g}(\bar{H} \bar{W}, \bar{H} \bar{Y})+\bar{c}\{\bar{g}(\bar{X}, \bar{Y}) \bar{g}(\bar{Z}, \bar{W}) \\
& -\bar{g}(\bar{Z}, \bar{X}) \bar{g}(\bar{Y}, \bar{W})+\eta(\bar{Z}) \eta(\bar{X}) \bar{g}(\bar{Y}, \bar{W}) \\
& -\eta(\bar{Y}) \eta(\bar{X}) \bar{g}(\bar{Z}, \bar{W})+\eta(\bar{Y}) \eta(\bar{W}) \bar{g}(\bar{Z}, \bar{X}) \\
& -\eta(\bar{Z}) \eta(\bar{W}) \bar{g}(\bar{Y}, \bar{X})+\bar{g}(\bar{\phi} \bar{Y}, \bar{X}) \bar{g}(\bar{\phi} \bar{Z}, \bar{W}) \\
& -\bar{g}(\bar{\phi} \bar{Z}, \bar{X}) \bar{g}(\bar{\phi} \bar{Y}, \bar{W}) \\
& -2 \bar{g}(\bar{\phi} \bar{Z}, \bar{Y}) \bar{g}(\bar{\phi} \bar{X}, \bar{W})\},
\end{aligned}
$$


Notice that $D_{0}$ and $\bar{\phi} \mathcal{S}$ are orthogonal and nondegenerate subbundles of $T M$ and that when $M$ is ascreen QGCR-null submanifold, we observe that

$$
\eta(X)=\eta(Z)=0, \quad \forall X \in \Gamma\left(D_{0}\right), Z \in \Gamma(\bar{\phi} \mathcal{S}) .
$$

Theorem 9. Let $\left(M, g, S(T M), S\left(T M^{\perp}\right)\right)$ be a totally umbilical or totally geodesic ascreen QGCR-null submanifold of an indefinite nearly cosymplectic space form $\bar{M}(\bar{c})$, of pointwise constant $\bar{\phi}$-sectional curvature $\bar{c}$, such that $D_{0}$ and $\bar{\phi} \mathcal{S}$ are space-like and parallel distributions with respect to $\nabla$. Then, $\bar{c} \geq 0$. Equality occurs when $\bar{M}(\bar{c})$ is an indefinite cosymplectic space form.

Proof. Let $X$ and $Z$ be vector fields in $D_{0}$ and $\bar{\phi} \mathcal{S}$, respectively. Replacing $\bar{W}$ with $\bar{\phi} X$ and $\bar{Y}$ with $\bar{\phi} Z$ in (36), we get

$$
\begin{aligned}
4 \bar{R}(X, \bar{\phi} X, Z, \bar{\phi} Z)= & \bar{g}\left(\left(\bar{\nabla}_{\bar{\phi} X} \bar{\phi}\right) Z,\left(\bar{\nabla}_{X} \bar{\phi}\right) \bar{\phi} Z\right) \\
& -\bar{g}\left(\left(\bar{\nabla}_{\bar{\phi} X} \bar{\phi}\right) \bar{\phi} Z,\left(\overline{\nabla_{X}} \bar{\phi}\right) Z\right) \\
& -2 \bar{g}\left(\left(\bar{\nabla}_{\bar{\phi} X} \bar{\phi}\right) X,\left(\bar{\nabla}_{\bar{\phi} Z} \bar{\phi}\right) Z\right) \\
& +\bar{g}(\bar{H} \bar{\phi} X, Z) \bar{g}(\bar{H} X, \bar{\phi} Z) \\
& -\bar{g}(\bar{H} \bar{\phi} X, \bar{\phi} Z) \bar{g}(\bar{H} X, Z) \\
& -2 \bar{g}(\bar{H} \bar{\phi} X, X) \bar{g}(\bar{H} \bar{\phi} Z, Z) \\
& -2 \bar{c} g(\bar{\phi} Z, \bar{\phi} Z) g(\bar{\phi} X, \bar{\phi} X) .
\end{aligned}
$$

Considering the first three terms on the right hand side of (38), we have

$$
\begin{aligned}
\bar{g} & \left(\left(\bar{\nabla}_{\bar{\phi} X} \bar{\phi}\right) Z,\left(\bar{\nabla}_{X} \bar{\phi}\right) \bar{\phi} Z\right) \\
& =-\bar{g}\left(\left(\bar{\nabla}_{Z} \bar{\phi}\right) \bar{\phi} X,\left(\bar{\nabla}_{X} \bar{\phi}\right) \bar{\phi} Z\right) .
\end{aligned}
$$

Applying (25) of Lemma 2 on (39) we derive

$$
\begin{aligned}
\bar{g} & \left(\left(\bar{\nabla}_{\bar{\phi} X} \bar{\phi}\right) Z,\left(\bar{\nabla}_{X} \bar{\phi}\right) \bar{\phi} Z\right) \\
= & -\bar{g}\left(\left(\bar{\nabla}_{Z} \bar{\phi}\right) \bar{\phi} X,\left(\bar{\nabla}_{X} \bar{\phi}\right) \bar{\phi} Z\right) \\
= & \bar{g}\left(\left(\bar{\nabla}_{X} \bar{\phi}\right) Z,\left(\bar{\nabla}_{X} \bar{\phi}\right) Z\right)-\bar{g}(\bar{\phi} Z, \bar{H} X)^{2} \\
& +\bar{g}(Z, \bar{H} X)^{2} .
\end{aligned}
$$

In a similar way, using (26) of Lemma 2, we get

$$
\begin{aligned}
& -\bar{g}\left(\left(\bar{\nabla}_{\bar{\phi} X} \bar{\phi}\right) \bar{\phi} Z,\left(\bar{\nabla}_{X} \bar{\phi}\right) Z\right) \\
& =\bar{g}\left(\left(\bar{\nabla}_{X} \bar{\phi}\right) Z,\left(\bar{\nabla}_{X} \bar{\phi}\right) Z\right), \\
& -2 \bar{g}\left(\left(\bar{\nabla}_{\bar{\phi} X} \bar{\phi}\right) X,\left(\bar{\nabla}_{\bar{\phi} Z} \bar{\phi}\right) Z\right)=0 .
\end{aligned}
$$

Now substituting (40) and (41) into (38), we get

$$
\begin{aligned}
4 \bar{R}(X, \bar{\phi} X, Z, \bar{\phi} Z)= & 2 \bar{g}\left(\left(\overline{\nabla_{X}} \bar{\phi}\right) Z,\left(\bar{\nabla}_{X} \bar{\phi}\right) Z\right) \\
& -\bar{g}(\bar{\phi} Z, \bar{H} X)^{2}+\bar{g}(Z, \bar{H} X)^{2} \\
& +\bar{g}(\bar{H} \bar{\phi} X, Z) \bar{g}(\bar{H} X, \bar{\phi} Z) \\
& -\bar{g}(\bar{H} \bar{\phi} X, \bar{\phi} Z) \bar{g}(\bar{H} X, Z) \\
& -2 \bar{g}(\bar{H} \bar{\phi} X, X) \bar{g}(\bar{H} \bar{\phi} Z, Z) \\
& -2 \bar{c} g(\bar{\phi} Z, \bar{\phi} Z) g(\bar{\phi} X, \bar{\phi} X),
\end{aligned}
$$

from which we obtain

$$
\begin{aligned}
2 \bar{R}(X, \bar{\phi} X, Z, \bar{\phi} Z)= & \bar{g}\left(\left(\bar{\nabla}_{X} \bar{\phi}\right) Z,\left(\bar{\nabla}_{X} \bar{\phi}\right) Z\right) \\
& +\bar{g}(Z, \bar{H} X)^{2} \\
& -\bar{c} g(Z, Z) g(X, X)
\end{aligned}
$$

Then using the facts that $D_{0}$ and $\bar{\phi} \mathcal{S}$ are space-like and parallel with respect to $\nabla$, we have $\left(\bar{\nabla}_{Z} \bar{\phi}\right) X=\left(\nabla_{Z} \bar{\phi}\right) X \in$ $\Gamma\left(D_{0}\right)$, and (43) reduces to

$$
\begin{aligned}
2 \bar{R}(X, \bar{\phi} X, Z, \bar{\phi} Z)= & \left\|\left(\nabla_{Z} \bar{\phi}\right) X\right\|^{2}+\bar{g}(Z, \bar{H} X)^{2} \\
& -\bar{c}\|X\|^{2}\|Z\|^{2},
\end{aligned}
$$

where $\|\cdot\|$ denotes the norm on $D_{0} \perp \bar{\phi} \mathcal{S}$ with respect to $g$.

On the other hand, if we set $\bar{W}=\bar{\phi} X$ and $\bar{Y}=\bar{\phi} Z$ in (14), we have

$$
\begin{aligned}
\bar{R}(X, \bar{\phi} X, Z, \bar{\phi} Z)= & \bar{g}\left(\left(\nabla_{X} h^{s}\right)(\bar{\phi} X, Z), \bar{\phi} Z\right) \\
& -\bar{g}\left(\left(\nabla_{\bar{\phi} X} h^{s}\right)(X, Z), \bar{\phi} Z\right),
\end{aligned}
$$

where

$$
\begin{aligned}
\left(\nabla_{X} h^{s}\right)(\bar{\phi} X, Z)= & \nabla_{X}^{s} h^{s}(\bar{\phi} X, Z)-h^{s}\left(\nabla_{X} \bar{\phi} X, Z\right) \\
& -h^{s}\left(\bar{\phi} X, \nabla_{X} Z\right)
\end{aligned}
$$

By the fact that $M$ is totally umbilical in $\bar{M}$, we have $h^{s}(\bar{\phi} X, Z)=0$. Thus using (16), (46) becomes

$$
\begin{aligned}
\left(\nabla_{X} h^{s}\right)(\bar{\phi} X, Z)= & -h^{s}\left(\nabla_{X} \bar{\phi} X, Z\right)-h^{s}\left(\bar{\phi} X, \nabla_{X} Z\right) \\
= & -g\left(\nabla_{X} \bar{\phi} X, Z\right) \mathscr{H}^{s} \\
& -g\left(\bar{\phi} X, \nabla_{X} Z\right) \mathscr{H}^{s} .
\end{aligned}
$$

Differentiating $\bar{g}(\bar{\phi} X, Z)=0$ covariantly with respect to $X$ and then applying $(7)$, we obtain

$$
g\left(\nabla_{X} \bar{\phi} X, Z\right)+g\left(\bar{\phi} X, \nabla_{X} Z\right)=0 .
$$


Substituting (48) into (47) gives

$$
\left(\nabla_{X} h^{s}\right)(\bar{\phi} X, Z)=0
$$

Similarly,

$$
\left(\nabla_{\bar{\phi} X} h^{s}\right)(X, Z)=0
$$

Then, substituting (49) and (50) into (45), we get

$$
\bar{R}(X, \bar{\phi} X, Z, \bar{\phi} Z)=0
$$

Substituting (51) into (44), gives

$$
\bar{c}\|X\|^{2}\|Z\|^{2}=\left\|\left(\nabla_{Z} \bar{\phi}\right) X\right\|^{2}+\bar{g}(Z, \bar{H} X)^{2} \geq 0
$$

which implies that $\bar{c} \geq 0$. When the ambient manifold is cosymplectic, then $\nabla \bar{\phi}=0$ and $d \eta=0$ [19] and in this case $\bar{c}=0$.

Example 10. Let $M$ be an ascreen QGCR-null submanifold in Example 7. Applying (7) and Koszul's formula (see [2]) to Example 7 we obtain

$$
\begin{aligned}
& h_{i}^{l}(X, Y)=0 \\
& \forall X, Y \in \Gamma(T M), \text { where } i=1,2,3, \\
& \epsilon_{4} h_{4}^{s}\left(X_{1}, X_{1}\right)=2, \\
& h_{4}^{s}(X, Y)=0, \\
& \forall X \neq X_{1}, Y \neq X_{1} .
\end{aligned}
$$

Using (12), (53), and $\epsilon_{4}=\bar{g}(W, W)=1+4\left(y^{5}\right)^{2}$, we also derive

$$
h\left(X_{1}, X_{1}\right)=\frac{2}{1+4\left(y^{5}\right)^{2}} W
$$

We remark that $M$ is not totally geodesic. From (54) and (15) we note that $M$ is totally umbilical with

$$
\mathscr{H}=\frac{2}{\left(1+4\left(y^{5}\right)^{2}\right)^{2}} W .
$$

By straightforward calculations we also have

$$
\begin{aligned}
& \nabla_{X_{1}} X_{1}=4 y^{5} X_{1}, \\
& \nabla_{X_{i}} X_{j}=0 \quad \forall i, j \neq 1 .
\end{aligned}
$$

Thus, $D_{0}$ and $\bar{\phi} \mathcal{S}$ are parallel distributions with respect to $\nabla$. Hence, $M$ satisfies Theorem 9 and $\bar{c}=0$.

Corollary 11. Let $\left(M, g, S(T M), S\left(T M^{\perp}\right)\right)$ be a totally umbilical or totally geodesic ascreen QGCR-null submanifold of an indefinite cosymplectic space form $\bar{M}(\bar{c})$ of pointwise constant $\bar{\phi}$-sectional curvature $\bar{c}$. Then, $\bar{c}=0$.
A null submanifold $M$ of a semi-Riemannian manifold $(\bar{M}, \bar{g})$ is called irrotational [3] if $\bar{\nabla}_{X} E \in \Gamma(T M)$, for any $E \in$ $\Gamma(\operatorname{Rad} T M)$ and $X \in \Gamma(T M)$. Equivalently, $M$ is irrotational if

$$
h^{l}(X, E)=h^{s}(X, E)=0,
$$

for all $X \in \Gamma(T M)$ and $E \in \Gamma(\operatorname{Rad} T M)$.

Theorem 12. Let $\left(M, g, S(T M), S\left(T M^{\perp}\right)\right)$ be an irrotational ascreen QGCR-null submanifold of an indefinite nearly cosymplectic space form $\bar{M}(c)$ of pointwise constant $\bar{\phi}$-sectional curvature $\bar{c}$. Then, $\bar{c} \leq 0$ or $\bar{c} \geq 0$. Equality holds when $\bar{M}(\bar{c})$ is an indefinite cosymplectic space form.

Proof. By setting $Y=E, Z=\bar{\phi} E$, and $W=E$ in (14), we get

$$
\begin{aligned}
\bar{R}(X, E, \bar{\phi} E, E)= & \bar{g} \\
& \left(\left(\nabla_{X} h^{l}\right)(E, \bar{\phi} E), E\right) \\
& -\bar{g}\left(\left(\nabla_{E} h^{l}\right)(X, \bar{\phi} E), E\right) \\
& +\bar{g}\left(\left(\nabla_{X} h^{s}\right)(E, \bar{\phi} E), E\right) \\
& -\bar{g}\left(\left(\nabla_{E} h^{s}\right)(X, \bar{\phi} E), E\right)
\end{aligned}
$$

for any $X \in \Gamma(T M)$ and $E \in \Gamma(\operatorname{Rad} T M)$. Then, using the fact that $M$ is irrotational, (58) reduces to

$$
\bar{R}(X, E, \bar{\phi} E, E)=0, \quad \forall X \in \Gamma(T M) .
$$

On the other hand, setting $\bar{Y}=\bar{W}=E$ and $\bar{Z}=\bar{\phi} E$ in (36) and simplifying, we get

$$
\begin{aligned}
\bar{R}(X, E, \bar{\phi} E, E)= & -3 \bar{g}\left(\left(\bar{\nabla}_{E} \bar{\phi}\right) \bar{\phi} E,\left(\bar{\nabla}_{E} \bar{\phi}\right) X\right) \\
& -\eta(E)^{2} \bar{g}(\bar{H} X, \bar{H} \bar{\phi} E) \\
& +4 \bar{c} \eta(E)^{2} \bar{g}(X, \bar{\phi} E) .
\end{aligned}
$$

Now, using (59) and (60), we get

$$
\begin{aligned}
4 \bar{c} \eta(E)^{2} \bar{g}(X, \bar{\phi} E)= & 3 \bar{g}\left(\left(\bar{\nabla}_{E} \bar{\phi}\right) \bar{\phi} E,\left(\bar{\nabla}_{E} \bar{\phi}\right) X\right) \\
& +\eta(E)^{2} \bar{g}(\bar{H} X, \bar{H} \bar{\phi} E) .
\end{aligned}
$$

Replacing $X$ with $\bar{\phi} E$ in (61) and using (25) of Lemma 2 to the resulting equation gives

$$
\bar{c} \eta(E)^{2} \bar{g}(\bar{\phi} E, \bar{\phi} E)=\eta(E)^{2} \bar{g}(\bar{H} \bar{\phi} E, \bar{H} \bar{\phi} E) .
$$

Since $M$ is ascreen QGCR-null submanifold, there exists $E \epsilon$ $\Gamma\left(D_{2}\right)$ such that $\eta(E)=b \neq 0$, and thus (62) simplifies to

$$
\bar{c}=-\frac{1}{b^{2}} \bar{g}(\bar{H} E, \bar{H} E)=\frac{1}{b^{2}} d \eta(E, \bar{H} E) .
$$

We observe that $\bar{c}=0$ if either $d \eta=0$ (i.e., $\bar{M}(c)$ is cosymplectic space form [19]) or $\bar{H} E$ is a null vector field. The second case implies that $\bar{H} E$ belongs to $\operatorname{Rad} T M$ or $l \operatorname{tr}(T M)$. If 
$\bar{H} E \in \Gamma(\operatorname{Rad} T M)$, then there exists a nonzero smooth function $\kappa$ such that $\bar{H} E=\kappa E$, for some arbitrary $E \in \Gamma(\operatorname{Rad} T M)$. Taking the $\bar{g}$-product of $\bar{H} E=\kappa E$ with $\xi$ leads to $0=\kappa \eta(E)$, from which $\eta(E)=0$. Since $M$ is ascreen QGCR-null submanifold, then, there is $E \in \Gamma\left(D_{2}\right)$ such that $\eta(E) \neq 0$, hence a contradiction. Similar reasoning can be applied if $\bar{H} E \in \Gamma(l \operatorname{tr}(T M))$. Therefore, $\bar{c}=0$ only if $\bar{H} E=0$ (i.e., $d \eta=$ 0 ) which occurs when $\bar{M}(c)$ is cosymplectic space form [19]. It turns out that $\bar{c} \leq 0$ or $\bar{c} \geq 0$ depending on whether $\bar{H} E$ is space-like or time-like vector field, respectively.

Corollary 13. Let $\left(M, g, S(T M), S\left(T M^{\perp}\right)\right)$ be an irrotational ascreen QGCR-null submanifold of an indefinite cosymplectic space form $\bar{M}(\bar{c})$ of pointwise constant $\bar{\phi}$-sectional curvature $\bar{c}$. Then, $\bar{c}=0$.

It is easy to see from (54) that $h^{l}(X, E)=h^{s}(X, E)=0$ and hence $M$ given in Example 10 is an irrotational ascreen QGCR-null submanifold of an indefinite cosymplectic space form $\bar{M}(\bar{c})$. As is proved in that example $\bar{c}=0$.

\section{Mixed Totally Geodesic QGCR-Null Submanifolds}

Definition 14. A QGCR-null submanifold of an indefinite nearly cosymplectic manifold $(\bar{M}, \bar{g})$ is called mixed totally geodesic if its second fundamental form, $h$, satisfies $h(X, Y)=$ 0 , for any $X \in \Gamma(D)$ and $Y \in \Gamma(\widehat{D})$.

We will need the following lemma in the next theorem.

Lemma 15. Let $\left(M, g, S(T M), S\left(T M^{\perp}\right)\right)$ be any 3-null proper ascreen QG CR-null submanifold of an indefinite nearly cosymplectic manifold $(\bar{M}, \bar{g})$. Then,

$$
2 \eta(E) \eta(N)=1,
$$

for any $E \in \Gamma\left(D_{2}\right)$ and $N \in \Gamma(\mathscr{L})$.

Proof. The proof follows from straightforward calculations using $\bar{g}(\xi, \xi)=1$ and $\xi=\eta(N) E+\eta(E) N$.

Theorem 16. Let $\left(M, g, S(T M), S\left(T M^{\perp}\right)\right)$ be a 3-null proper ascreen QGCR-null submanifold of an indefinite nearly cosymplectic manifold $(\bar{M}, \bar{g})$. Then, $M$ is mixed totally geodesic if and only if $h_{\alpha}^{s}(X, Y)=0$ and $A_{E_{i}}^{*} X=0$, for all $X \in \Gamma(D)$, $Y \in \Gamma(\widehat{D}), W_{\alpha} \in \Gamma\left(S\left(T M^{\perp}\right)\right)$, and $E_{i} \in \Gamma(\operatorname{Rad} T M)$.

Proof. By the definition of ascreen QGCR-null submanifold, $M$ is mixed geodesic if

$$
\bar{g}\left(h(X, Y), W_{\alpha}\right)=\bar{g}\left(h(X, Y), E_{i}\right)=0,
$$

for all $X \in \Gamma(D), Y \in \Gamma(\widehat{D}), W_{\alpha} \in \Gamma\left(S\left(T M^{\perp}\right)\right)$, and $E_{i} \in$ $\Gamma(\operatorname{Rad} T M)$. Now, by virtue of (12) and the first equation of (65), we have

$$
0=\bar{g}\left(h(X, Y), W_{\alpha}\right)=\epsilon_{\alpha} h_{\alpha}^{s}(X, Y),
$$

from which $h_{\alpha}^{s}(X, Y)=0$, since $\epsilon_{\alpha} \neq 0$. On the other hand, using (65), (7), and (11) we derive

$$
\begin{aligned}
\bar{g}\left(h(X, Y), E_{i}\right) & =\bar{g}\left(\bar{\nabla}_{X} Y, E_{i}\right)=-\bar{g}\left(Y, \bar{\nabla}_{X} E_{i}\right) \\
& =g\left(Y, A_{E_{i}}^{*} X\right)=0 .
\end{aligned}
$$

Since $D=D_{0} \perp D_{1}$ and $\widehat{D}=\left\{D_{2} \perp \bar{\phi} D_{2}\right\} \oplus \bar{D}$, we observe that $A_{E_{i}}^{*} X \notin \Gamma\left(\bar{\phi} D_{2}\right)$ or $\bar{\phi} \mathscr{L}$. In fact, suppose that $A_{E_{i}}^{*} X \notin \Gamma\left(\bar{\phi} D_{2}\right)$; then there exists a nonvanishing smooth function $\ell$ such that $A_{E_{i}}^{*} X=\ell \bar{\phi} E$, for $E \in \Gamma\left(D_{2}\right)$. Thus,

$$
0=g\left(Y, A_{E_{i}}^{*} X\right)=\lg (Y, \bar{\phi} E), \quad \forall Y \in \Gamma(\widehat{D}) .
$$

Taking $Y=\bar{\phi} N$ in (68), where $N \in \Gamma(\mathscr{L})$ and using Lemma 15, we have

$$
\begin{aligned}
0 & =g\left(Y, A_{E_{i}}^{*} X\right)=\lg (\bar{\phi} N, \bar{\phi} E)=\ell(1-\eta(E) \eta(N)) \\
& =\frac{1}{2} \ell,
\end{aligned}
$$

which is a contradiction, since $\ell \neq 0$. Hence $A_{E_{i}}^{*} X \notin \Gamma\left(\bar{\phi} D_{2} \oplus\right.$ $\bar{\phi} \mathscr{L})$. Moreover, $A_{E_{i}}^{*} X \notin \Gamma(\bar{\phi} \mathcal{S})$ since if $A_{E_{i}}^{*} X \in \Gamma(\bar{\phi} \mathcal{S})$, then there is a nonvanishing smooth function $\omega$ such that $A_{E_{i}}^{*} X=$ $\omega \bar{\phi} W_{\alpha}$. Taking the $\bar{g}$-product of this equation with respect to $Y=\bar{\phi} W_{\alpha}$ and using the fact that $\eta\left(W_{\alpha}\right)=0$, we get

$$
\begin{aligned}
0 & =g\left(Y, A_{E_{i}}^{*} X\right)=\omega \bar{g}\left(\bar{\phi} W_{\alpha}, \bar{\phi} W_{\alpha}\right)=\omega \bar{g}\left(W_{\alpha}, W_{\alpha}\right) \\
& =\omega \epsilon_{\alpha},
\end{aligned}
$$

which is a contradiction, since $\epsilon_{\alpha} \neq 0$ and $\omega \neq 0$. Hence, $A_{E_{i}}^{*} X \notin \Gamma\left(\left\{\bar{\phi} D_{2} \oplus \bar{\phi} \mathscr{L}\right\} \perp \bar{\phi} \mathcal{S}\right)$, which implies that $A_{E_{i}}^{*} X \in$ $\Gamma\left(D_{0}\right)$. Since $A_{E_{i}}^{*} X \in \Gamma\left(D_{0}\right)$, then the nondegeneracy of $D_{0}$ implies that there exists some $Z \in \Gamma\left(D_{0}\right)$ such that $g\left(A_{E_{i}}^{*} X\right.$, $Z) \neq 0$. But using (11) and (7), together with the fact that $M$ is mixed geodesic, we derive

$$
\begin{aligned}
g\left(A_{E_{i}}^{*} X, Z\right) & =-g\left(\nabla_{X} E_{i}, Z\right)=\bar{g}\left(E_{i}, \bar{\nabla}_{X} Z\right) \\
& =\bar{g}\left(E_{i}, \nabla_{X} Z\right)=0,
\end{aligned}
$$

which is a contradiction. Thus $A_{E_{i}}^{*} X \notin \Gamma\left(\left\{\bar{\phi} D_{2} \oplus \bar{\phi} \mathscr{L}\right\} \perp \bar{\phi} \mathcal{S} \perp\right.$ $D_{0}$ ); that is, $A_{E_{i}}^{*} X=0$. The converse is obvious.

Corollary 17. Let ( $\left.M, g, S(T M), S\left(T M^{\perp}\right)\right)$ be a proper ascreen QGCR-null submanifold of an indefinite nearly cosymplectic manifold $(\bar{M}, \bar{g})$. Then, if $M$ is mixed totally geodesic then $h_{i}^{l}\left(X, E_{i}\right)=0$ and $\varphi_{\alpha i}(X)=0$, for all $X \in \Gamma(D)$ and $E_{i} \in \Gamma\left(D_{2}\right)$.

Definition 18. A QGCR-null submanifold of an indefinite nearly cosymplectic manifold $(\bar{M}, \bar{g})$ is called $D$-totally geodesic if its second fundamental form $h$ satisfies $h(X, Y)=0$, $\forall X, Y \in \Gamma(D)$. 
Since $M$ is ascreen QGCR-null submanifold, we have $\bar{g}(X, \xi)=0$, for all $X \in \Gamma(D)$. Applying $\bar{\nabla}_{Y}$ to $\bar{g}(X, \xi)=0$ we get

$$
\eta\left(\bar{\nabla}_{Y} X\right)=-\bar{g}\left(X, \bar{\nabla}_{Y} \xi\right)=\bar{g}(X, \bar{H} Y)
$$

Interchanging $X$ and $Y$ in (72) and then adding the resulting equation to $(72)$ gives

$$
\eta\left(\bar{\nabla}_{X} Y\right)+\eta\left(\bar{\nabla}_{Y} X\right)=\bar{g}(Y, \bar{H} X)+\bar{g}(X, \bar{H} Y)=0 .
$$

Theorem 19. Let $\left(M, g, S(T M), S\left(T M^{\perp}\right)\right)$ be a proper ascreen QGCR-null submanifold of an indefinite nearly cosymplectic manifold $(\bar{M}, \bar{g})$. Then, $M$ is D-totally geodesic if and only if $\bar{\phi} h^{l}(X, \bar{\phi} E)$ and $\bar{\phi} h^{s}(X, \bar{\phi} W)$, respectively, have no components along $l \operatorname{tr}(T M)$ and $S\left(T M^{\perp}\right)$, while both $\nabla_{X} \bar{\phi} E$ and $\nabla_{X} \bar{\phi} W \notin$ $\Gamma\left(D_{0}\right)$ for all $X \in \Gamma(D), E \in \Gamma(\operatorname{Rad} T M)$, and $W \in \Gamma(\mathcal{S})$.

Proof. By the definition of ascreen QGCR-null submanifold, $M$ is $D$ geodesic if and only if $\bar{g}(h(X, Y), E)=\bar{g}(h(X$, $Y), W)=0$, for all $X, Y \in \Gamma(D), W_{\alpha} \in \Gamma\left(S\left(T M^{\perp}\right)\right)$, and $E \in \Gamma(\operatorname{Rad} T M)$.

Using (7) and (18), we derive

$$
\begin{aligned}
\bar{g}(h(X, Y), E)= & \bar{g}\left(\bar{\nabla}_{X} Y, E\right) \\
= & \bar{g}\left(\bar{\phi} \bar{\nabla}_{X} Y, \bar{\phi} E\right) \\
& -\bar{g}\left(Y, \bar{\nabla}_{X} \xi\right) \bar{g}(E, \xi),
\end{aligned}
$$

from which when we apply (20) we get

$$
\begin{aligned}
\bar{g}(h(X, Y), E)= & \bar{g}\left(\bar{\phi} \bar{\nabla}_{X} Y, \bar{\phi} E\right) \\
& +\bar{g}(Y, \bar{H} X) \bar{g}(E, \xi) .
\end{aligned}
$$

Interchanging $X$ and $Y$ in (75) and considering the fact that $h$ is symmetric we get

$$
\begin{aligned}
\bar{g}(h(X, Y), E)= & \bar{g}\left(\bar{\phi} \bar{\nabla}_{Y} X, \bar{\phi} E\right) \\
& +\bar{g}(X, \bar{H} Y) \bar{g}(E, \xi) .
\end{aligned}
$$

Summing (75) and (76) and then applying (73), we have

$$
2 \bar{g}(h(X, Y), E)=\bar{g}\left(\bar{\phi} \bar{\nabla}_{X} Y, \bar{\phi} E\right)+\bar{g}\left(\bar{\phi} \bar{\nabla}_{Y} X, \bar{\phi} E\right) .
$$

Now, applying the nearly cosymplectic condition in (19) to (77) leads to

$$
2 \bar{g}(h(X, Y), E)=\bar{g}\left(\bar{\nabla}_{X} \bar{\phi} Y, \bar{\phi} E\right)+\bar{g}\left(\bar{\nabla}_{Y} \bar{\phi} X, \bar{\phi} E\right) .
$$

From (78) and (7) we derive

$$
\begin{aligned}
2 \bar{g}(h(X, Y), E)= & \bar{g}\left(\bar{\nabla}_{X} \bar{\phi} Y, \bar{\phi} E\right)+\bar{g}\left(\bar{\nabla}_{Y} \bar{\phi} X, \bar{\phi} E\right) \\
& -\bar{g}(\bar{\phi} Y, h(X, \bar{\phi} E)) \\
& -\bar{g}(\bar{\phi} X, h(Y, \bar{\phi} E)) .
\end{aligned}
$$

If we let $X, Y \in \Gamma\left(D_{1}\right)$ in (79), we obtain

$$
\begin{aligned}
2 \bar{g}(h(X, Y), E)= & \bar{g}(Y, \bar{\phi} h(X, \bar{\phi} E)) \\
& +\bar{g}(X, \bar{\phi} h(Y, \bar{\phi} E)) .
\end{aligned}
$$

On the other hand, for $X, Y \in \Gamma\left(D_{0}\right)$, we get

$$
2 \bar{g}(h(X, Y), E)=-\bar{g}\left(\bar{\phi} Y, \nabla_{X} \bar{\phi} E\right)-\bar{g}\left(\bar{\phi} X, \nabla_{Y} \bar{\phi} E\right) .
$$

It is easy to see from (80) and (81) that if $\bar{\phi} h(X, \bar{\phi} E) \notin$ $\Gamma(l \operatorname{tr}(T M))$ and $\nabla_{X} \bar{\phi} E \notin \Gamma\left(D_{0}\right)$, then $\bar{g}(h(X, Y), E)=0$. The other assertions follows in the same way. The converse is obvious.

Corollary 20. Let $\left(M, g, S(T M), S\left(T M^{\perp}\right)\right)$ be a proper ascreen QGCR-null submanifold of an indefinite nearly cosymplectic manifold $(\bar{M}, \bar{g})$. If $M$ is D-totally geodesic then $\nabla_{X}^{*} \bar{\phi} E$ and $\nabla_{X}^{*} \bar{\phi} W \notin \Gamma\left(D_{0}\right)$, for all $X \in \Gamma(D), E \in \Gamma\left(D_{2}\right)$, and $W \in$ $\Gamma(\mathcal{S})$.

Corollary 21. Let $\left(M, g, S(T M), S\left(T M^{\perp}\right)\right)$ be a proper ascreen QGCR-null submanifold of an indefinite nearly cosymplectic manifold $(\bar{M}, \bar{g})$. If $M$ is D-totally geodesic, then $D$ defines a totally geodesic foliation in $M$.

\section{Competing Interests}

The authors declare that there is no conflict of interests regarding the publication of this paper.

\section{Acknowledgments}

Samuel Ssekajja extends his sincere gratitude to the African Institute of Mathematical Sciences (AIMS) and the Simon Foundation through the RGSM-Network project, for their financial support during this research. Finally, both authors are grateful to the referee for his/her valuable comments and suggestions that helped them improve the paper.

\section{References}

[1] D. N. Kupeli, Singuler Semi-riemannian Geometry, vol. 366 of Mathematics and Its Applications, Kluwer Academic, 1996.

[2] K. L. Duggal and A. Bejancu, Lightlike Submanifolds of SemiRiemannian Manifolds and Applications, vol. 364 of Mathematics and Its Applications, Kluwer Academic Publishers, Dordrecht, The Netherlands, 1996.

[3] K. L. Duggal and B. Sahin, Differential Geometry of Lightlike Submanifolds, Mathematics and Its Applications, Kluwer Academic, 2008.

[4] K. L. Duggal and D. H. Jin, Null Curves and Hypersurfaces of Semi-Riemannian Manifolds, World Scientific Publishing, 2007.

[5] K. L. Duggal and B. Sahin, "Generalized Cauchy-Riemann lightlike submanifolds of indefinite Sasakian manifolds," Acta Mathematica Hungarica, vol. 122, no. 1-2, pp. 45-58, 2009.

[6] K. L. Duggal and D. J. Jin, "Generic lightlike submanifolds of indefinite Sasakian manifolds," International Electronic Journal of Geometry, vol. 5, no. 1, pp. 108-119, 2012. 
[7] D. H. Jin, "Geometry of lightlike hypersurfaces of an indefinite Sasakian manifold," Indian Journal of Pure and Applied Mathematics, vol. 41, no. 4, pp. 569-581, 2010.

[8] F. Massamba, "Totally contact umbilical lightlike hypersurfaces of indefinite Sasakian manifolds," Kodai Mathematical Journal, vol. 31, no. 3, pp. 338-358, 2008.

[9] F. Massamba, "On semi-parallel lightlike hypersurfaces of indefinite Kenmotsu manifolds," Journal of Geometry, vol. 95, no. 1-2, pp. 73-89, 2009.

[10] F. Massamba, "On lightlike geometry in indefinite Kenmotsu manifolds," Mathematica Slovaca, vol. 62, no. 2, pp. 315-344, 2012.

[11] F. Massamba, "Symmetries of null geometry in indefinite Kenmotsu manifolds," Mediterranean Journal of Mathematics, vol. 10, no. 2, pp. 1079-1099, 2013.

[12] C. Calin, Contributions to geometry of CR-submanifold [Ph.D. thesis], University of Iași, Iași, Romania, 1998.

[13] K. L. Duggal and B. Sahin, "Lightlike submanifolds of indefinite Sasakian manifolds," International Journal of Mathematics and Mathematical Sciences, vol. 2007, Article ID 57585, 21 pages, 2007.

[14] K. Yano and M. Kon, CR submanifolds of Kaehlerian and Sasakian Manifolds, vol. 30 of Progress in Mathematics, Birkhäuser, Boston, Mass, USA, 1983.

[15] F. Massamba and S. Ssekajja, "Quasi generalized CR-lightlike submanifolds of indefinite nearly Sasakian manifolds," Arabian Journal of Mathematics, vol. 5, no. 2, pp. 87-101, 2016.

[16] D. H. Jin, "Ascreen lightlike hypersurfaces of indefinite Sasakian manifolds," Journal of the Korean Society of Mathematical Education Series B, vol. 20, no. 1, pp. 25-35, 2013.

[17] M. Ahmad and M. D. Siddiqui, "On a Nearly Sasakian manifold with a semi-symmetric semi-metric connection," International Journal of Mathematical Analysis, vol. 4, no. 35, pp. 1725-1732, 2010.

[18] D. E. Blair, D. K. Showers, and K. Yano, "Nearly Sasakian structures," Kodai Mathematical Seminar Reports, vol. 27, no. 1-2, pp. 175-180, 1976.

[19] D. E. Blair, Contact Manifolds in Riemannian Geometry, vol. 509 of Lecture Notes in Mathematics, Springer, Berlin, Germany, 1976.

[20] B. Cappelletti-Montano and G. Dileo, "Nearly Sasakian geometry and SU(2)-structures," Annali di Matematica Pura ed Applicata. Series IV, vol. 195, no. 3, pp. 897-922, 2016.

[21] H. Endo, "On the curvature tensor of nearly cosymplectic manifolds of constant $\Phi$-sectional curvature," Analele Stiintifice ale Universitatii Al I Cuza din Iasi-Matematica, vol. 51, no. 2, pp. 439-454, 2005.

[22] E. Loubeau and E. Vergara-Diaz, "The harmonicity of nearly cosymplectic structures," Transactions of the American Mathematical Society, vol. 367, no. 8, pp. 5301-5327, 2015. 


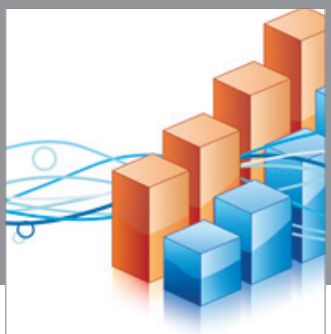

Advances in

Operations Research

vatem alat4

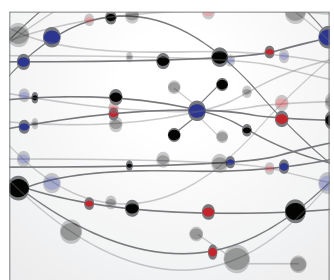

\section{The Scientific} World Journal
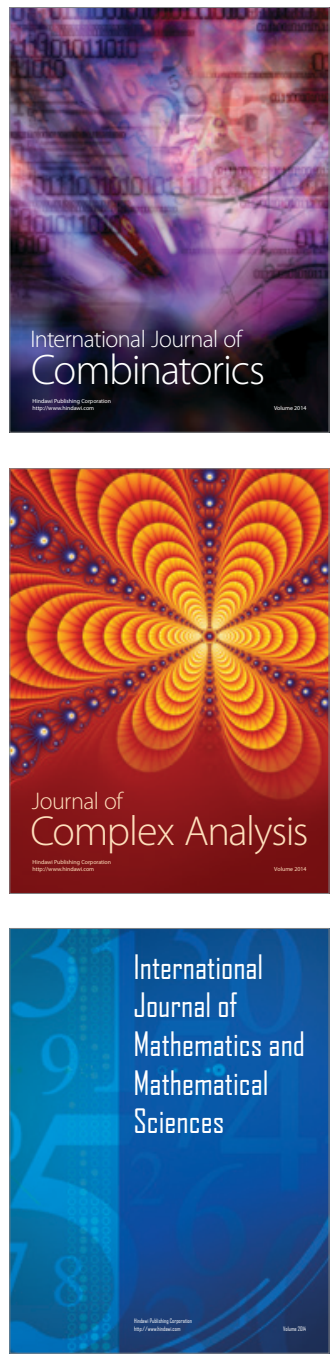
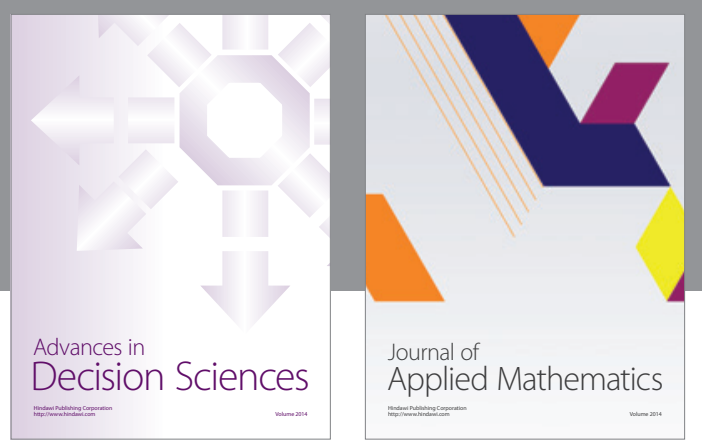

Algebra

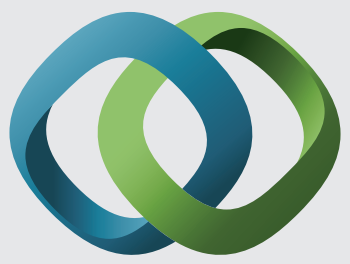

\section{Hindawi}

Submit your manuscripts at

http://www.hindawi.com
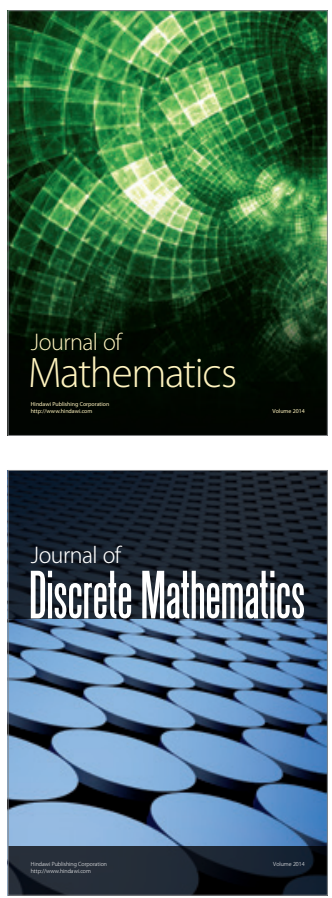

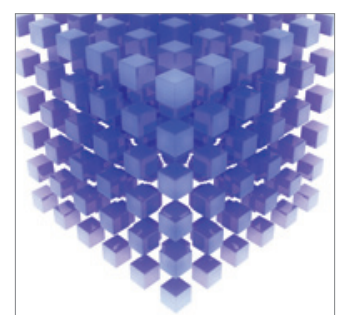

Mathematical Problems in Engineering
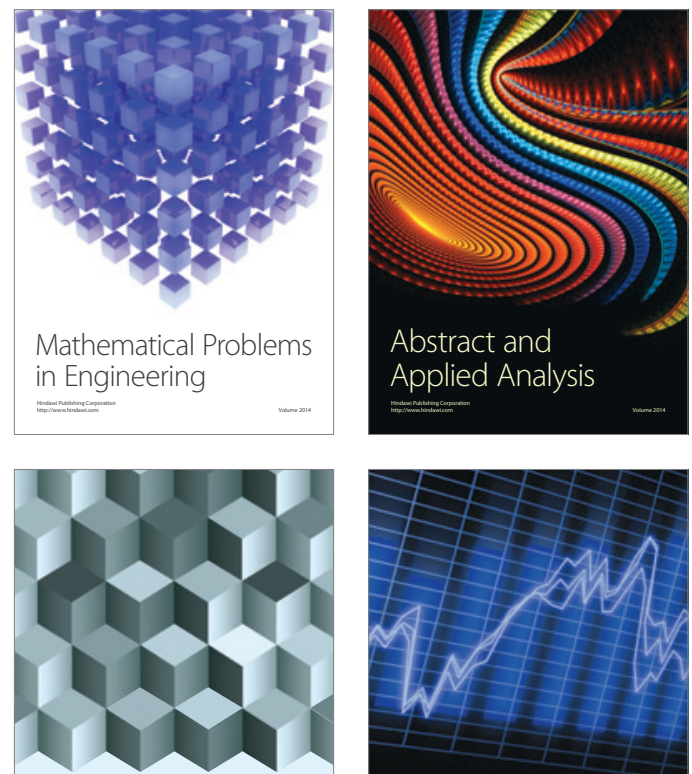

Journal of

Function Spaces

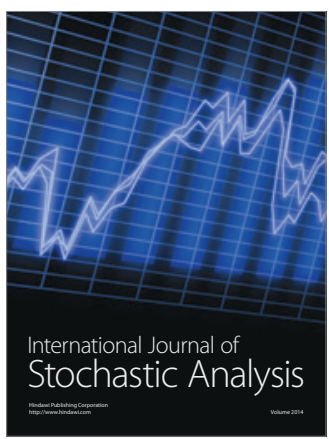

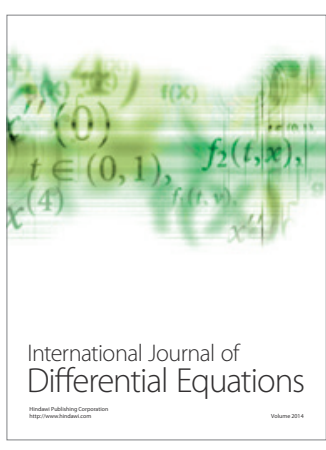
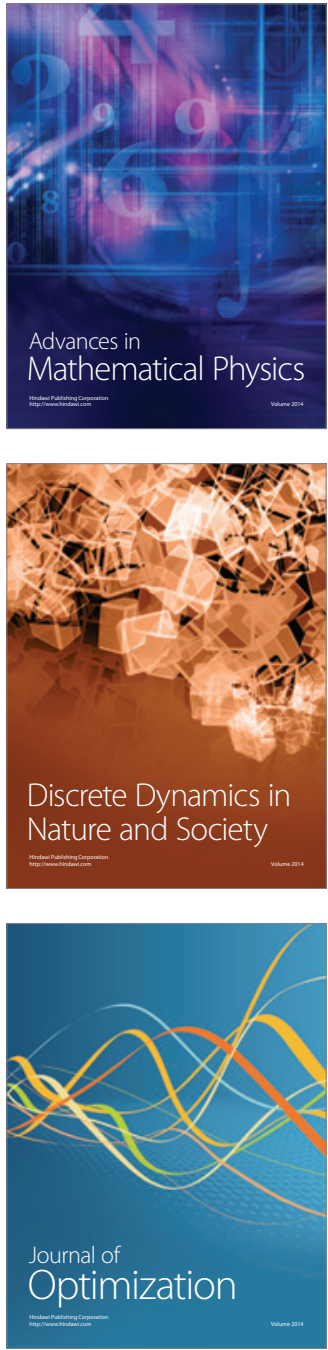